\title{
Tangible Pointer Trailing and Signal Appreciation for Electronic Video Gaming
}

\author{
N. Venkateswaran \\ Department of ECE \\ SSN College of Engineering \\ Kalavakkam, Chennai, India
}

\author{
P.S. Jagadeesh Kumar \\ Department of CSE \\ MIT, Aurangabad \\ Maharashtra, India
}

\author{
L.M.I. Leo Joseph \\ Department of ECE \\ SR Engineering College \\ Warangal, Telangana, India
}

\begin{abstract}
The probable critique guidelines the mechanism of a computer game based on pointer signal appreciation. The aspiration entails the ceiling of tangible retort and unconfined milieus. The paper largess a here and now system to trail and discern hand gestures to be integrated with the electronic video game. This algorithm is centered on three focal stride outs; hand dissection, hand trailing and gesture appreciation from hand topography. However, the hand dissection step routines, color nod owing to the distinctive color tenets of human skin; it's infrangibly chattels and its reckoning easiness. To inhibit booboos from hand dissection, the ensuing stride, hand trailing is ancillary. The trailing technique is artistic and condescending persistent speed model using the pixel tagging methodology. The trailing strategy intangibles, profuse hand topography that is fed to a determinate formal categorizer which pinpoints the hand conformation. The hand can be labeled into any of the four gesticulation curricula or in any of the four crusade directions. To end with, by means of the system's enactment fallout, the consumption of the recommended system in an electronic game environment is publicized.
\end{abstract}

\section{General Terms}

Pointer Trailing, Signal Appreciation, Hand Topography

\section{Keywords}

Electronic Video Game, Computer Communication

\section{INTRODUCTION}

At present, the preponderance of the Human Computer Interaction (HCI) is accustomed on physical maneuvers such as mouse, game pad, keyboard and joystick. In modern eons, there is an emergent concern in approaches founded on computational vision owing to its capability to diagnose human movements in a usual manner [1-3]. These techniques custom the images attained from a camera or from a highfidelity duo of cameras as input. The central aim of this algorithm is to ration hand pattern in every stage instantly. This procedure grafts concurrent for compact dimension hand and clenches paramount image [4]. To expedite this practice, countless gesture appreciation proffers to the usage of inimitably tinted scarves or symbols on hands. Furthermore, expending a meticulous context styles it probable to confine the hand competently in real-time. These circumstances inflict constraints on the user and on the border outfit. Here are evaded precise elucidations that necessitate colored gloves and a well-ordered context as an earlier constraint to this application [5]. It functions for dissimilar people, devoid of any supplement on them and for impulsive circumstances. The scheme habits imageries from a stumpy price web camera positioned in the lead of the enclosure, where the familiar gesticulations turn as the input for 3D computer game. Thus, the players, instead of corresponding buttons, necessarily use diverse gestures that the system should identify. This data enhances the intricacy that the reaction time needs be debauched. Users have, not to escalate a momentary delay concerning the instant they accomplish a gesture and the instant the system retorts. Consequently, the algorithm must deliver concurrent concert for conservative processor [6].

The pointer trailing and appreciation algorithm do not bump into this obligation and incongruous for visual crossing point. For example, Element filtering-based procedures can sustain numerous propositions at the same time to vigorously trail the hands. Recently, some mechanism has been unfilled for tumbling the intricacy of element filters, perhaps, expending a deterministic progression to assist the haphazard pursuit [7]. Though, these systems solitarily grind in real-time for a compact dimension hand; the hand grips furthermost of the spitting image. In this paper, projected a simultaneous nonintrusive hand trailing and gesture appreciation scheme. In the following segments are elucidating the projected scheme alienated in three leading strides [8]. The first phase is hand dissection, which encompasses the hand, devise to be traced. Appropriate to craft this practice, it is plausible to custom contours, but they diverge momentously through the regular gesticulation of hand. Consequently, skin-shade is chosen as the hand chattel. The skin-shade is an idiosyncratic nod of hands and it is apathetic to gage and gyration. The subsequent pace is to trail the location and alignment of the hand to thwart faults in the dissection stage. A pixel centered trailing for the time-based appraisal of the hand ceremonial is throwaway [9]. In the preceding step, the sketchy, hand status to extort numerous hand descriptions to describe a deterministic progression of signal appreciation is worn. In the end, the anticipated method illustrates that the system performance assessment works fine in an unimpeded environment.

\section{HAND DISSECTION}

The hand ought to be restricted in the image and dissected from the ambience before detection. Color is the certain nod as of its computational ease, its impervious properties about the hand silhouette configurations and attributable to the individual skin-color idiosyncratic values. Moreover, the postulation that color can be utilized as a reminder to identify faces and hand in numerous periodical. In this relevance, the hand dissection has been conceded by means of a squat calculative costly scheme that does well in concurrent. The process is fashioned in a probabilistic form of the skin-color pixel allocation. Subsequently, it is obligatory to replicate the skin-color of the punter's hand. The user lays ingredients of his hand in the learning plaza. The pixels constrained in this locale will be utilized for the sophistication of the sculpt. Then, the particular pixels are distorted as of the RGB to the 
HSL for intriguing the chroma data: hue and saturation. Beside, bumping into two major exertions in the hand dissection step; it has been resolved in the processing stage. The primary one is that individual skin tone ideals are very close to red color, specifically, their significance is on the brink of $2 \pi$ radians, so it is hard to study the distribution owing to the hue bony nature that can fabricate model on both confines. Fissure is once the infiltration ideals are near to 0 , since then the hue is unbalanced and can be the rationale for false detections. This can be evaded by forsaking infiltration ideals near 0 as shown in Fig 1.

Once dispensation stage has finished, the hue and saturation ideals of every selected pixel are employed to deduce the model. As an outcome of trying and evaluating stage with some arithmetical sculpt such as a concoction of Gaussians or distinct histograms, the finest effects have been conquered through Gaussian model. The ideals for the constraints of the Gaussian model such as mean and covariance matrix are calculated from the taster set by implementing the standard maximum likelihood technique. Once they are established, the probability that of the new pixel can be calculated. Ultimately, the projected scheme obtains the dribble depiction of the hand applying a linked mechanism algorithm to the likelihood image, which clusters pixels into the similar blob.

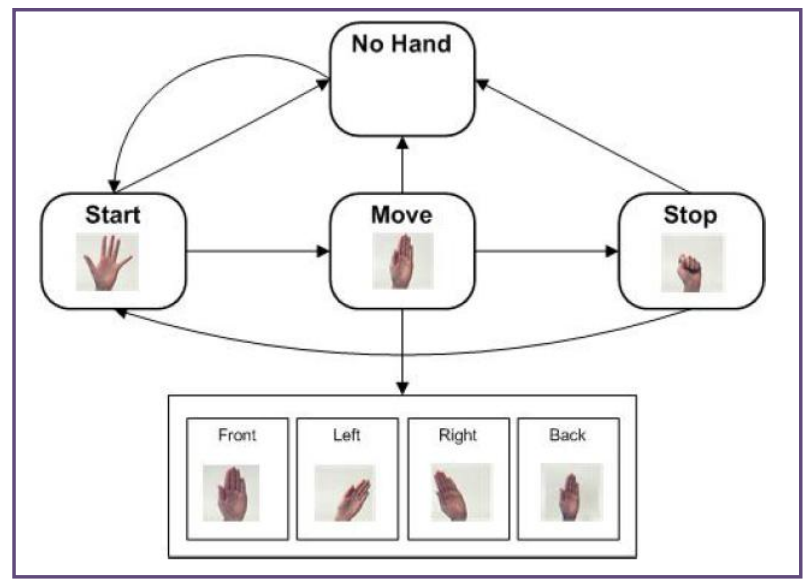

Fig 1: Hand Dissection

\section{HAND TRAILING}

USB cameras are renowned for the low feature images they generate. This reality can root errors in the hand dissection procedure. In turn to craft the relevance vigorously, tracking algorithm is auxiliary. This algorithm attempts to uphold and broadcast the hand position in time. Primarily, propositions of the hand position are constructed from the hand pose in t time, for time $t+1$ a straightforward second-order autoregressive progression to the location constituent is inferred. These articulate a vibrating replica of steady velocity. Subsequently, if alleged that at time t, M dribbles have been professed, where each dribble commune to any situate of its associated color pixels, the trailing procedure has to situate the relative amid the hand premise and the surveillance at occasions. Consecutively to handle with this predicament, a ballpark figure was definite to the expanse from the pixel, $x=(x, y) r$, to the theory. This expanse can be observed as the estimation of the detachment from a point in the $2 \mathrm{D}$ emancipation to a regularized ellipse. Regularized means centered in starting point and not swiveled. From the expanse description it twirls out that its significance is equivalent or beneath 0 as shown in Fig 2.

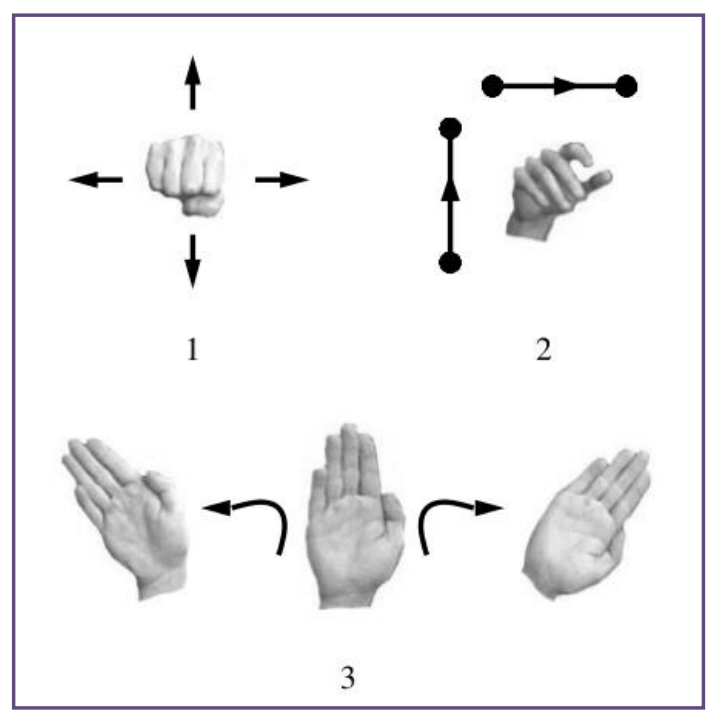

Fig 2: Hand Trailing

\section{SIGNAL APPRECIATION}

The gesticulation alphabet utilizes four hand gestures and four hand instructions to facilitate the users' necessities. The hand gestures match to a completely unbolt hand with alienated fingers, a released hand with fingers joined, a fist and the last gesture emerges when the hand is not discernible, infraction or entirely, in the camera's turf of outlook. These signals are identified as Start, Move, Stop correspondingly. Moreover, when the consumer is in the Move signal, he can lug out a Left, Right, Front and Back actions. For the Left and Right actions, the consumer will turn his wrist to the left or right. From the Front and Back actions, the hand will get nearer to or auxiliary position of the camera. The procedure of signal appreciation begins when the hand signals are placed ahead of the camera turf of outlook and the hand is in the Start signal, to be precise, hand with alienated fingers. For evading fast hand signal amends that were not anticipated, every modification ought to be reserved flat for 5 frames; if not the pointer signal does not transform from the preceding predictable signal. To attain signal appreciation, the hand state estimate is used in the trailing process. In addition to the hand's shape and the hand's curved hull, a series of outline points between two consecutive curved hull vertices is premeditated. This series structures the ostensible convexity blemish and it is likely to compute the profundity of the $i^{\text {th }}$ convexity blemish. From this nadir, some valuable uniqueness of the hand shape can be consequential by the profundity middling.

Fig 3 illustrates the start and the end point of the $i^{\text {th }}$ convexity blemish, the profundity, id, is the detachment from the furthermost point of the convensity blemish of the curved hull section. The prime step of the signal appreciation process is to sculpt the Start signal. The middling of the profundity of the convexity blemish of an unbolt hand with alienated fingers is better-quality than when the hand is not discernible. This trait is worn for discriminating the subsequent hand signal shift: from Stop to Start; from Start to Move; and from No-Hand to Start. Conversely, it is essential to calculate the Start signal feature. Once the consumer is properly located in the camera turf of outlook with the hand extended unbolt for erudition of 
his skin-color, and the scheme also calculates the Start signal feature for the $\mathrm{n}$ initial frames.

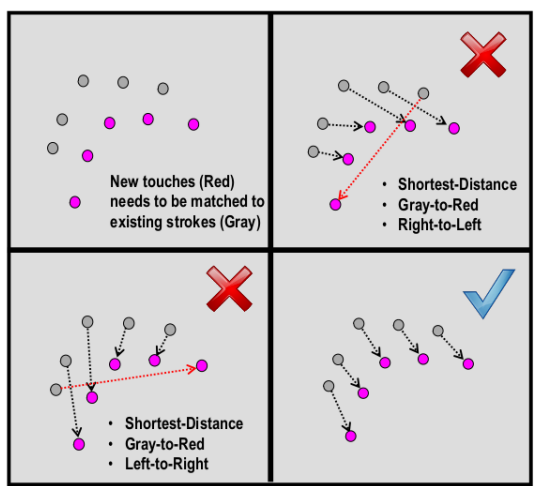

Fig 3: Convexity blemish and the profundity

Subsequent to the identification of the Start signal, the most probable legitimate signal alters is the Move signal. After that, if the present hand profundity is less than the system goes to the Move hand signal, the current hand signal is Move the hand commands will be aided: Front, Back, Left and Right. If the consumer does not desire to move in any bearing, he should situate his hand in Move state. For the first time that the Move signal materializes, the system reckons the Move signal trait that is the middling of the approximated area of the hand for $\mathrm{n}$ successive frames. For distinguishing the Left and Right instructions, the deliberated angle of the integral ellipse is dilapidated. To avert redundant jitter effects in this track, a predefined invariable is distinct. Followed by, if the angle of the ellipse that demarcates the hand, persuades, Left direction will be placed. If the angle of the ellipse that demarcates the hand is discontented, Right direction will be placed. For scheming the Front and Back directions and for returning to the Move signal, the hand must not be swiveled and the Move signal trait is used for distinguishing these actions. If thrives the hand direction will be Front. The Back direction will be attained if back. The Stop signal will be documented by means of the ellipse axis. When the hand is in a fist, the integral ellipse is approximately like a circle; $\mathrm{m}$ and $\mathrm{M}$ are virtually the same, which is a predefined constant recognized during the performance evaluation system. To conclude, the No-Hand state will emerge when the structure does not perceive the hand, the size of the identified hand is not adequate or when the hand is at the frontier of the camera turf of the outlook. The subsequent feasible hand state will be the Start gesture and it will be noticed by the transition process from Stop to Start signal. Various examples for signal fruition and the standard gesture results can be seen in Fig 4. It is very imperative to achieve a correct erudition of the skin-color. If not, some tribulations with the discovery and the signal appreciation can be encountered. One of the central problems with the exercise of the submission is the hand control to sustain it in the camera's turf of outlook and exclusive poignant, that confines the capture area. This crisis has been revealed to wane with customer training.

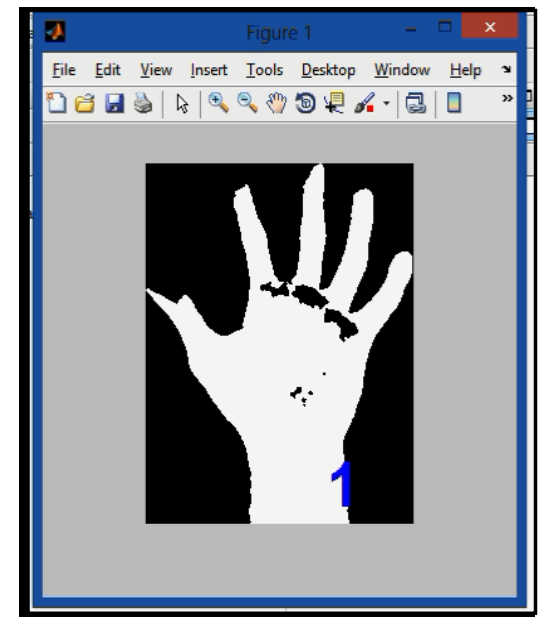

Fig 4: Signal Appreciation

\section{PERFORMANCE EVALUATION}

In this segment, the precision of pointer trailing and the signal appreciation algorithm is portrayed. The relevance has been executed in MS Visual C++ using the Open CV libraries and MATLAB Simulink environment. The appliance has been experienced on an Intel Core i5 Processor with 8GB RAM. The imagery has been incarcerated by means of a Logitech Webcam. The camera affords $1020 \times 1240$ images at a confine and dispensation rate of 50 frames per second. For the recital assessment of the pointer trailing and signal appreciation, the arrangement has been experienced on a set of 50 consumers. Each user has performed a delineated set of 70 actions and therefore 690 signals are evaluated for the relevant outcomes. It is normal to believe that the scheme's correctness will be premeditated on calculating the performance of preferred consumer actions to manage the video gaming. This progression integrated all the appliance probable conditions and shifts. Fig 5 shows the performance evaluation results. These results are symbolized by means of a two-dimensional matrix with the functional condition as columns and the number of emergences of the signal as rows. The columns are matched for each signal: the first column is the number of trials of the signal that has been rightly recognized; the second column is the total number of times that the gesture has been conceded. As it can be seen in Fig 6,7 and 8, the hand appreciation signal mechanizes good for a $99 \%$ of the crates.

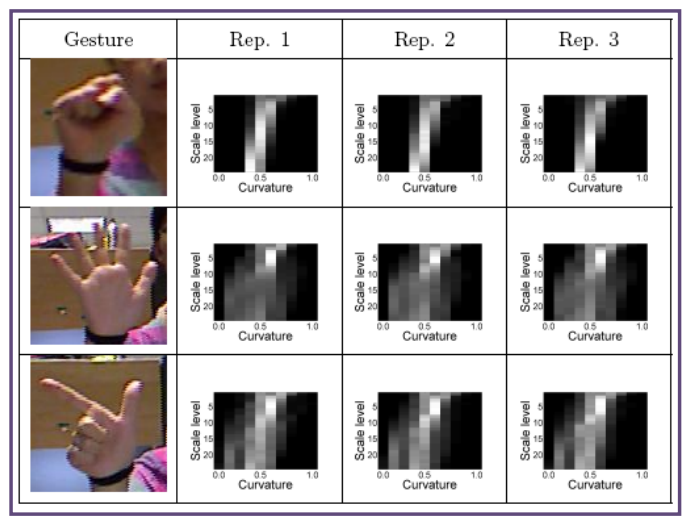

Fig 5: Performance Evaluation results 


\section{CONCLUSION}

In this critique, compassionated a tangible pointer trailing and signal appreciation for human computer interface, indorsing the locale of electronic video gaming. The projected exertion is plinth on hand dissection, hand trailing, signal appreciation from wringed hand budge. The concert insinuation fallouts have publicized that this economical brim can be browbeaten by the consumers to surrogate habitual interface allegory. The tryouts have inveterate that incessant training of the consumer fallouts in privileged dexterity and better recital.

\section{REFERENCES}

[1] P.S.Jagadeesh Kumar, N.Venkateswaran, R Vijaya Prakah, Pittala Manasa. 2016. Pragmatic Delineation of Multifaceted Vibrant Scheme for Imprudent Animatronics. International Journal of Electrical and Computer Engineering.

[2] P.S.Jagadeesh Kumar. 2016. Bespoked Xography for Pointer Trailing and Signal Appreciation of Computer Games. 18th International Conference on Computer Vision Theory and Applications, Tokyo, Japan. World Academy of Science, Engineering and Technology.

[3] N.Venkateswaran, P.S.Jagadeesh Kumar. 2016. Detonation of Artificial Intelligence for Virtual Reality based 3D Game Design. SAI IntelliSys2016 Conference, IEEE, London, UK.

[4] P.S.Jagadeesh Kumar, S.Meenakshmi Sundaram. 2015. Understanding the Contrive Confront of 3D Video Game Augmentation and Contraption. International Journal of Computer Science and Information Security, USA.
[5] Monika Sarve, Deepak Khatri. 2014. 3D game design and development of 3D shooter game using Xna. International Conference on Industrial Automation and Computing.

[6] Siddharth, Manjusha. 2014. Single and Multiple Hand Gesture Recognition Systems: A Comparative Analysis. International Journal of Intelligent Systems and Applications.

[7] Prashan Premaratne, Sabooh Ajaz, Malin Premaratne. 2013. Hand gesture tracking and recognition system using Lucas-Kanade algorithms for control of consumer electronics. Journal of Neurocomputing.

[8] Piyush Kumar, Jyoti Verma, Shitala Prasad. 2012. A Hand Data Glove: A Wearable Real-Time Device for Human Computer Interaction. International Journal of Advanced Science and Technology.

[9] Dejan Chandra Gope. 2012. Hand Tracking and Hand Gesture Recognition for Human Computer Interaction. American Academic and Scholarly Research Journal.

[10] Noor Adnan Ibraheem, Rafiqul Zaman Khan. 2012. Survey on Various Gesture Recognition Technologies and Techniques. International Journal of Computer Applications.

[11] Tin Hninn. 2009. Real-Time Hand Tracking and Gesture Recognition System Using Neural Networks. World Academy of Science, Engineering and Technology.

\section{APPENDIX}

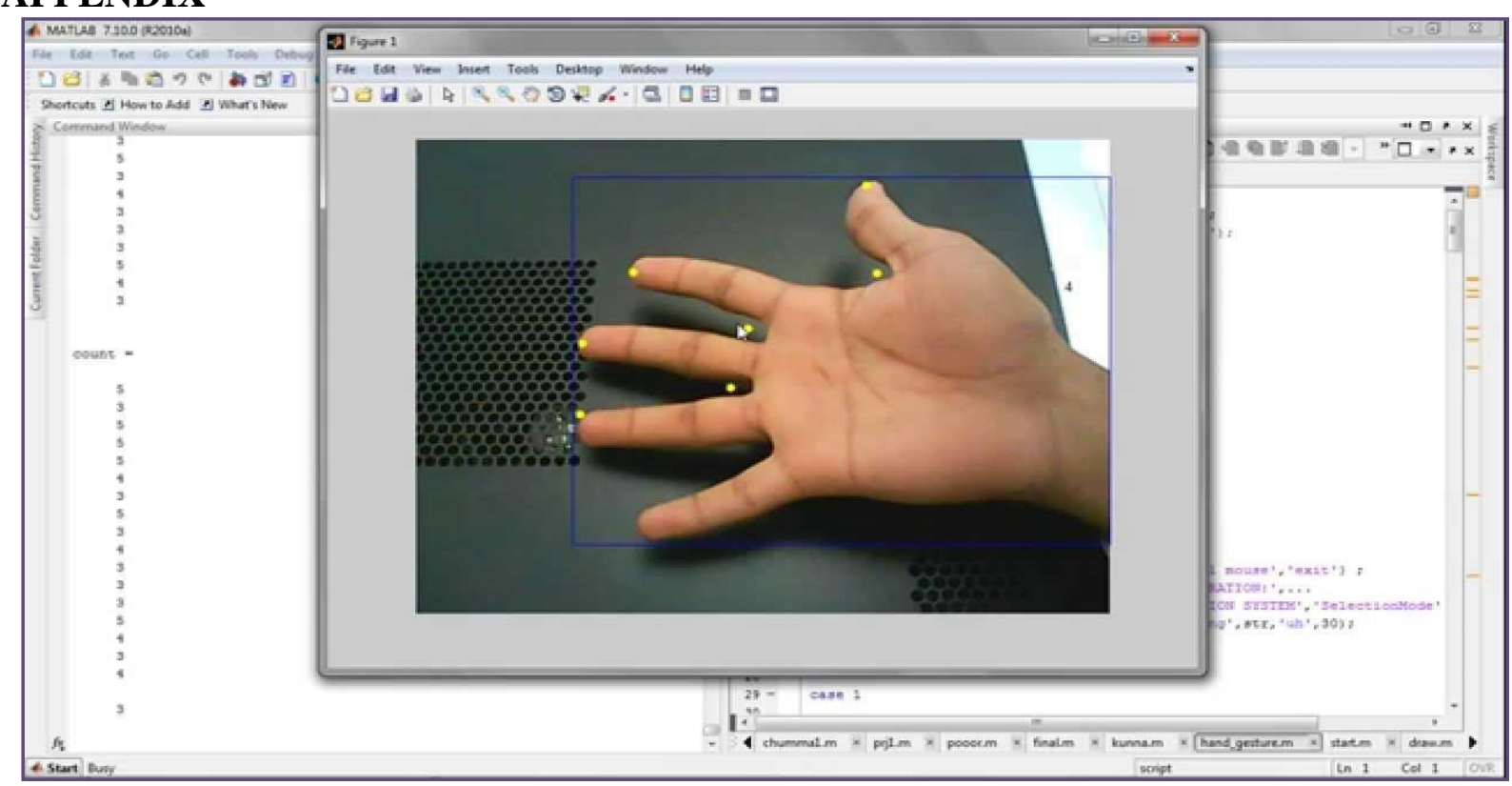

Fig 6: Tangible pointer trailing using MATLAb Simulink environment 


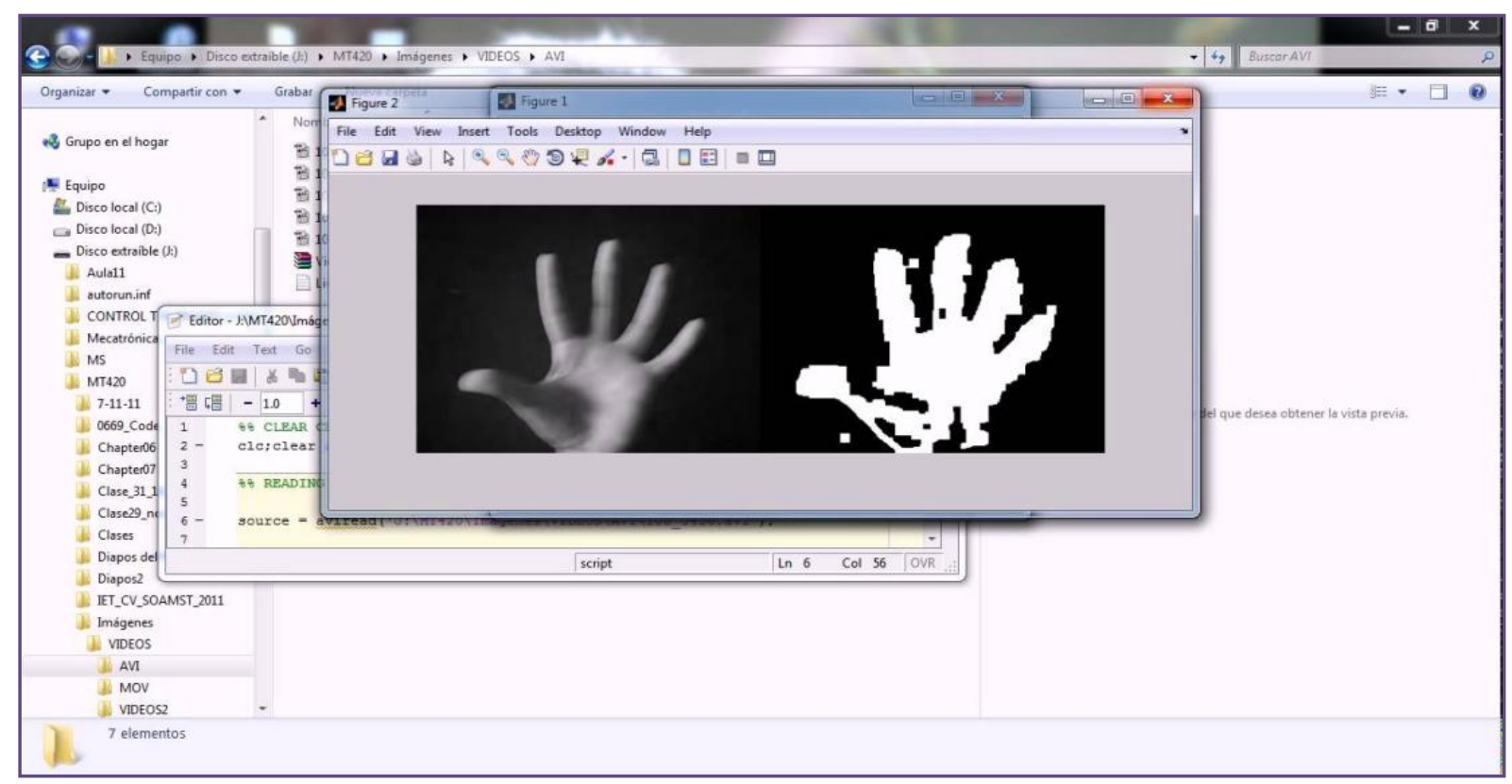

Fig 7: Signal Appreciation using MATLAb Simulink environment

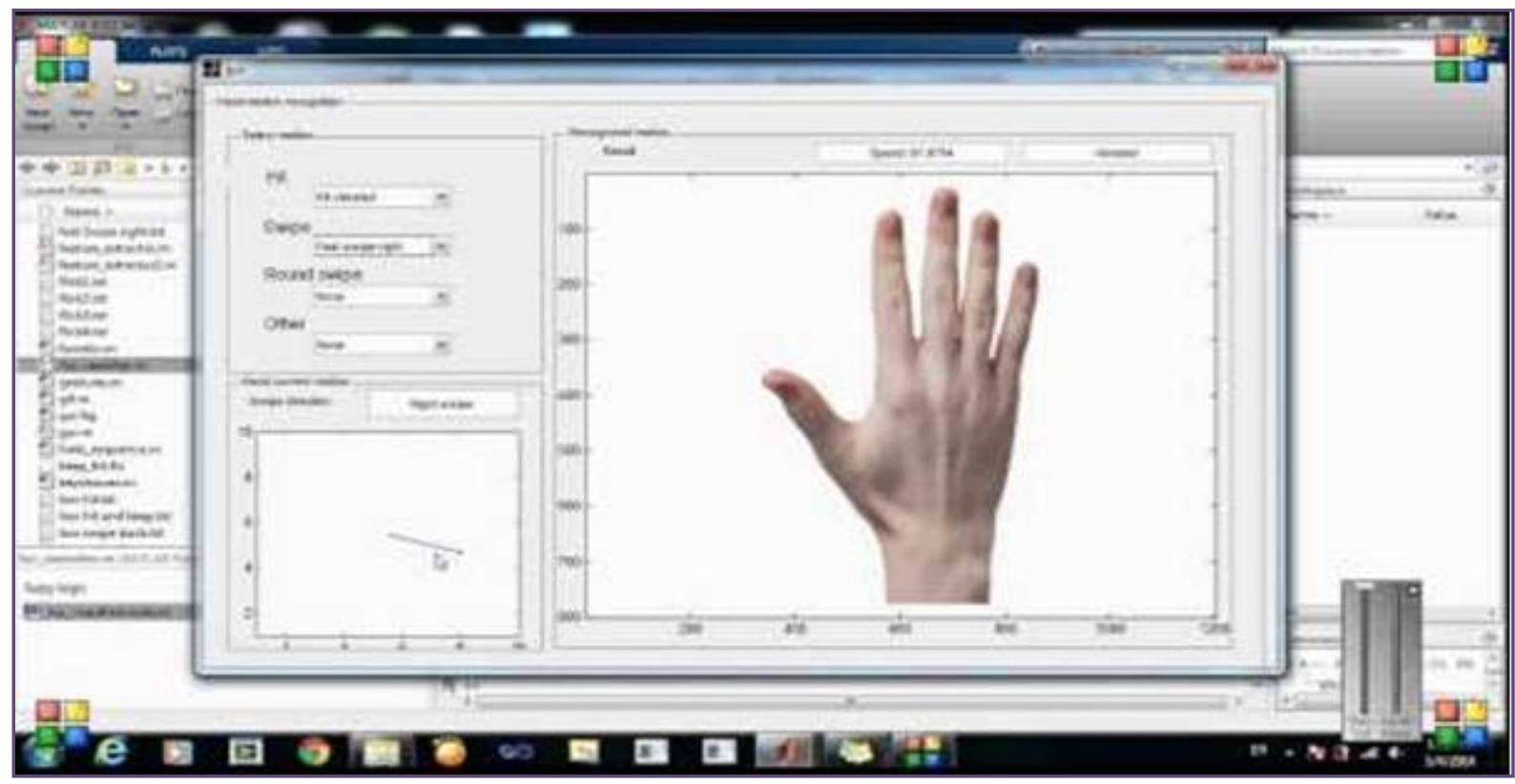

Fig 8: Hand Trailing using MS Visual C++ Open CV libraries 